\title{
DeFi, Not So Decentralized: The Measured Distribution of Voting Rights
}

\author{
Barbereau, Tom \\ University of Luxembourg \\ tom.barbereau@uni.lu \\ ORCID: 0000-0002-8554-0991
}

\author{
Rieger, Alexander \\ University of Luxembourg \\ alexander.rieger@uni.lu \\ ORCID: 0000-0001-7996-4678
}

\author{
Smethurst, Reilly \\ University of Luxembourg \\ reilly.smethurst@uni.lu \\ ORCID: 0000-0002-6191-475X
}

\author{
Papageorgiou, Orestis \\ University of Luxembourg \\ orestis.papageorgiou@uni.lu \\ ORCID: 0000-0002-2245-5079
}

\author{
Fridgen, Gilbert \\ University of Luxembourg \\ gilbert.fridgen@uni.lu \\ ORCID: 0000-0001-7037-4807
}

\begin{abstract}
Bitcoin and Ethereum are frequently promoted as decentralized, but developers and academics question their actual decentralization. This motivates further experiments with public permissionless blockchains to achieve decentralization along technical, economic, and political lines. The distribution of tokenized voting rights aims for political decentralization. Tokenized voting rights achieved notoriety within the nascent field of decentralized finance (DeFi) in 2020. As an alternative to centralized crypto-asset exchanges and lending platforms (owned by companies like Coinbase and Celsius), DeFi developers typically create noncustodial projects that are not majority-owned or managed by legal entities. Holders of tokenized voting rights can instead govern DeFi projects. To scrutinize DeFi's distributed governance strategies, we conducted a multiple-case study of non-custodial, Ethereum-based DeFi projects: Uniswap, Maker, SushiSwap, Yearn Finance, and UMA. Our findings are novel and surprising: quantitative evaluations of DeFi's distributed governance strategies reveal a failure to achieve political decentralization.
\end{abstract}

\section{Introduction}

In January 2021, Uniswap became the first noncustodial crypto-asset exchange to reach USD \$100 billion trading volume - a significant event for the nascent field of decentralized finance (DeFi). DeFi projects are typically governed by online communities of anonymous stakeholders rather than companies or public institutions. Instead of registered shareholders' voting rights, many DeFi projects involve tokenized voting rights, and they offer unregulated financial services that are based on crypto-assets instead of sovereign currencies. For DeFi, the meaning of 'decentralized' thus entails independence from regulated companies, investor registration processes, and central banks $[1,2,3]$. The descriptor 'decentralized' does not necessarily denote a uniform distribution of capital or voting power [4]. Political decentralization is anything but guaranteed.

In 1856, the historian Alexis de Tocqueville [5] investigated the fall of the French aristocracy and the subsequent dissolution of power. Contrary to the revolutionaries' expectations, the abolition of feudalism in France at the end of the $18^{\text {th }}$ century did not effectively limit the power of all central authorities. Authority putatively transferred from the aristocracy to the people, yet there soon emerged a powerful bureaucratic system known as the Première République. "When a nation abolishes aristocracy," De Tocqueville concluded, "centralization follows as a matter of course: everything tends toward unity of power, and it requires no small contrivance to maintain divisions of authority."

De Tocqueville's maxim retains an air of truth in the era of putatively decentralized networks like Bitcoin and Ethereum. The ideals of decentralization that inspired the invention of Bitcoin and Ethereum are undermined by tendencies towards socio-political centralization $[6$, $7,8,9,10]$ and technical centralization $[11,12]$. Developers and active members of crypto-asset communities are aware of these tendencies; hence they propose novel, experimental strategies to achieve decentralization. DeFi projects are the most prominent decentralization experiments from late 2020 [2]. Many entail strategies to distribute tradable voting rights tokens (also known as governance tokens) for the sake of political decentralization [1]. 
DeFi's voting rights tokens are speculative cryptoassets, not backed by anything physical [13]. They enable token-holders to iteratively revise a project's rules, parameters, and features. A broad range of DeFi projects issue voting rights tokens, from non-custodial crypto-asset exchanges to derivatives platforms [1]. Maker - a non-custodial crypto-asset lending platform initiated the trend with its MKR token.

Uniswap is the largest non-custodial, Ethereumbased exchange. Uniswap issued its voting rights token (UNI) in September 2020 via a retrospective airdrop. The airdrop rewarded anonymous wallet owners that interacted with the Uniswap exchange in the past (by swapping tokens or providing liquidity). UNI tokenholders today govern the Uniswap exchange. They have the right to vote on proposals that pertain to treasury funds and changes to the exchange's rules; and, if they wish, they can sell their UNI tokens on various cryptoasset exchanges, or they can purchase more UNI tokens to increase their voting power. As of 9 June 2021, the UNI token is in the top 10 list of crypto-assets (sorted by market capitalization), and it sells for more than four times its initial price of $\$ 5$.

While academic research has addressed cryptoassets, Bitcoin, Ethereum, algorithmic governance, and the various meanings of 'decentralization,' noncustodial DeFi projects are comparatively uncharted territory, and tokenized voting rights issued by $\mathrm{DeFi}$ projects are an under-researched topic. It is not yet known if De Tocqueville's pessimism about decentralization remains valid for DeFi's distributed voting rights; hence we formulated the following research questions. How did major, Ethereum-based DeFi projects distribute voting rights? What are the outcomes of the different distribution strategies?

We conducted an exploratory, multiple-case study in response to these questions [14]. We selected as cases five non-custodial, Ethereum-based DeFi projects that include tradable voting rights tokens: Uniswap, Maker, SushiSwap, Yearn Finance, and UMA. Our case selection covers four major DeFi categories [1]: lending (Maker), exchanges (Uniswap and SushiSwap), assets (Yearn Finance), and derivatives (UMA). We selected two exchanges instead of just one exchange, because SushiSwap essentially began as a clone of Uniswap.

We followed a mixed methods approach [15], which is recognized for its ability to discover the unexpected. First, we qualitatively analyzed project documentation that details the properties and the distribution strategy for each case's tokenized voting rights. We then quantitatively examined the "physical" artifact [14], based on methods proposed by Gochhayat et al. [11].
We specifically used metrics that quantify the degree of uniformity for each case's distribution of voting rights tokens.

\section{Background: The promise of 'decentralization'}

Bitcoin is the fastest asset to reach a $\$ 1$ trillion market capitalization [16]. It achieved this feat with no legal entity attached to it as a manager or a majority owner. Bitcoin's pseudonymous creator, Satoshi Nakamoto, established a finite supply (21 million units of BTC) that cannot be altered by a central bank or any other financial authority [17]. Bitcoin transactions are not registered on servers owned by a single bank, financial institution, or consortium; the Bitcoin ledger is instead distributed across a global computing network that consists of voluntary participants [18]. In comparison with sovereign currencies, physical commodities, and other tangible assets, Bitcoin is difficult to regulate or subject to capital controls [19, 20].

Social scientists often interpret Bitcoin as a symptom of widespread distrust in both financial and political institutions following the 2007-2008 global financial crisis $[6,8]$. The raw hex data from Bitcoin's genesis block, mined by Nakamoto on 3 January 2009, contains within it The Times' daily headline: "Chancellor on brink of second bailout for banks." Sociologists class Bitcoin's early adopters as fundamentalist libertarians, many of whom conceive 'decentralization' as a set of economic and political ideals [10, 21]. De Filippi \& Loveluck [6] traced the Bitcoin community's conception of 'decentralization' to "Friedrich Hayek's and Milton Friedman's ambition to end the monopoly of nation-states."

The ideals of decentralization enjoy a broad appeal among libertarians. For the libertarian Right, decentralization's principal mission is to end the oligopoly of banks and tax authorities, and to create a market without costly intermediaries and powerful regulators. For the libertarian Left, decentralization's principal mission is to abolish centralized monetary policy and State capital controls $[6,22]$. There is no necessary link, however, between the Bitcoin community's ideals of decentralization and an actualized, uniform distribution of capital or voting power within the Bitcoin network [9, 23]. As Kostakis \& Giotitsas [4] put it, "in theory you have equipotential individuals (that is, everyone can potentially participate in a project), but in practice what one gets is concentrated capital and centralized governance." 


\subsection{Bitcoin's re-centralization and unregulated store of value}

On the one hand, Bitcoin exhibits non-uniform distributions of capital, governance authority, and computing power, which together imply a failure to achieve decentralization. On the other hand, the Bitcoin network poses a challenge to centralized legal authorities and regulators, which means it is indeed 'decentralized' in the anti-establishment, libertarian sense.

According to the Gini index, capital (BTC) is distributed less evenly in the Bitcoin network than it is in economies like South Africa or Brazil [23, 24]. The 2015 block-size debate and subsequent revision to the Bitcoin protocol rules demonstrated the concentrated governance power held by the Bitcoin Core Developers and the Lead Developer [6,9]. In addition, costly mining hardware (application-specific integrated circuits, or ASICs), owned by a sophisticated minority of Bitcoin miners, offers evidence of concentrated computing power $[11,12,25]$. Today, one private company in China, Bitmain, dominates the global ASIC production industry [26], and it owns the Bitcoin mining pools AntPool and BTC.com. These pools together comprise over $20 \%$ of the Bitcoin network's hash rate (Figure 1 - data retrieved from btc.com on 7 June 2021).

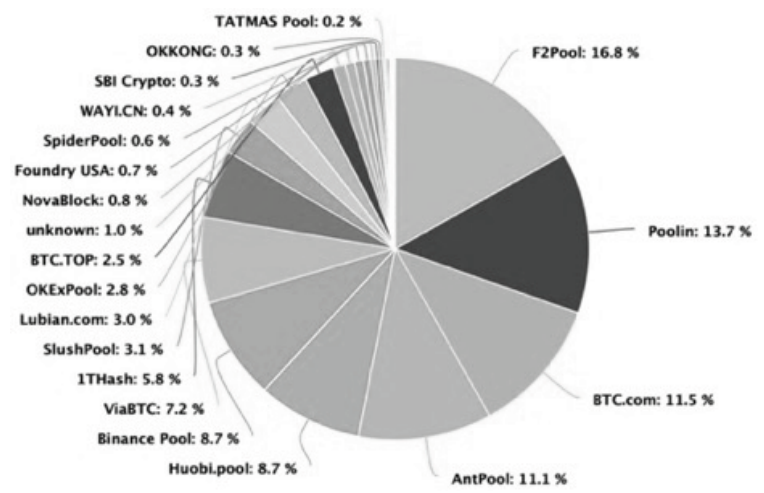

Figure 1. Mining pools dominate the hash rate

The Bitcoin network does, however, successfully manifest this libertarian ideal of decentralization: it is uncontrollable or unregulatable. Since the global Bitcoin network is diffuse and not wholly grounded in any specific jurisdiction, it is a challenge for regulators $[27,28,29]$. The Bitcoin network is also a challenge to regulate because there is no legal entity attached to it as a manager or a majority owner. In legal terms, Bitcoin can thus be classified as an "unincorporated distributed ledger system" [3]. Lawmakers also struggle to define the legal status of Bitcoin's store of value (BTC) [19]; hence regulatory responses to BTC are incoherent and autarkic [27].

\subsection{Ethereum's unregulated contracts, non- liable organizations, and centralized traits}

Ethereum introduced its own, unregulated store of value $(\mathrm{ETH})$ in 2015; but, more importantly for the present study, Ethereum also introduced unregulated chain code, referred to as smart contracts [30]. Smart contracts do not require an intermediary authority to execute them - another variation on the theme of 'decentralization' [10]. Smart contracts can bind together members of decentralized autonomous organizations (DAOs), and they can also assign voting rights [31].

A DAO is a protocol that 'lives' on the Internet and consists of formalized rules [31]. If truly decentralized, no single entity (including a DAO's creator) can alter, reverse, or control a DAO once it is deployed on the Internet. A DAO is virtually unregulatable and nonliable: it simply executes its pre-determined set of 'ifthen' rules [2, 32]. If a DeFi project delegates management duties to an online community instead of a legal entity, then it is effectively a DAO $[1,2]$.

Like Bitcoin, the Ethereum network is sometimes criticized for its compromised or failed decentralization $[11,25]$. Researchers cast doubt upon Ethereum's ability to abolish corporate intermediaries and usher in a "decentralized world" [25, 33]. In 2016, Ethereum's core developers exercised autocratic powers [8, 34, 35] and established a new fork of the blockchain, in response to a disastrous hack. De Tocqueville's maxim thus applies to Ethereum's governance as well: "centralization follows as a matter of course" [5].

\subsection{DeFi projects not bound to regulated companies: centralized, once again?}

The year 2020 witnessed multi-billion-dollar trading volumes for Ethereum-based financial services projects that do not rely on regulated, custodial intermediaries such as banks, brokerages, or company-governed exchanges [1, 3, 36]. These projects - commonly categorized as DeFi - include non-custodial cryptoasset exchanges, lending platforms, and derivatives platforms [1]. DeFi projects are often governed by a non-liable DAO rather than a legal entity [2, 3]; hence they are distinct from centralized crypto-asset 
exchanges and lending platforms owned by regulated companies like Coinbase and Celsius.

DeFi exchanges like Uniswap and SushiSwap allow participants to vote for proposed changes to rules, parameters, and features, so long as the participants purchase or else earn voting rights tokens (via contributions to the exchange) [13]. This phenomenon can be interpreted in at least two distinct ways: (a) governance is decentralized, because decision-making power is distributed among a community of tokenholders (and therefore not held by a centralized company or institution), and (b) governance is timocratic, since voting rights are exclusively attributed to 'property owners' (in this case, token-holders). The second interpretation is especially pertinent to SushiSwap. On 7 September 2020, a small group of individuals held the majority of voting rights tokens, and they successfully seized control of the exchange [37]. De Tocqueville's maxim, once again, rings true.

A DeFi project named Yearn Finance pre-empted outcomes like this, hence it deployed a refined fair launch strategy. Fair launch strategies vary from case to case; but in general, a fair launch does not allocate a large portion of pre-mined (spontaneously generated) tokens to developers or early investors and advisors [38]. A fair launch is premised on the following assumption: if a significant portion of voting rights tokens are distributed to privileged insiders, then it is difficult to describe a project's governance as decentralized. The fair launch is a sub-topic of Ethereum tokenomics and token distribution strategies [13, 39, 40].

Yearn Finance's fair launch strategy, deployed in July 2020, distributed none of the project's voting rights tokens (YFI) to developers and early investors. Yearn Finance's fair launch did not abolish timocracy, since only YFI token-holders possess voting rights. What its fair launch did, specifically, is remove the distinction between 'insiders' (that are allocated voting rights by default) and 'outsiders' (that must either purchase voting rights on exchanges, provide liquidity, or contribute work to the project). Other DeFi projects, such as Beefy Finance, Harvest Finance, and YAM, later deployed fair launch strategies as well.

DeFi's various fair launch strategies indicate new avenues for research into algorithmic governance and political decentralization, set against a backdrop of timocracy, re-centralization, and concentrated powers.

\section{Methods}

To understand DeFi's tokenized voting rights and to evaluate the success of various distribution strategies, we conducted an exploratory, multiple-case study [14] with a mixed methods approach [15]. For the sake of consistent quantitative data extraction, we only chose DeFi projects that utilize Ethereum, and we only used Etherscan. We limited our study to Ethereum, because it has more daily transactions than rival ledgers like the Binance Smart Chain, Solana, and Terra. We selected distributions of voting rights tokens that have a market capitalization of more than $\$ 1$ billion. Four of our cases cover distinct DeFi categories [1]: lending (Maker), exchanges (Uniswap), assets (Yearn Finance), and derivatives (UMA). We added a fifth case, SushiSwap, because of its close relation to Uniswap plus the fact that SushiSwap issued voting rights tokens before Uniswap did. The initial version of SushiSwap, dated 26 August 2020 , derived most of its smart contract code from Uniswap Version 2 [41]. At the time, there was one major distinction between the two exchanges: holders of voting rights tokens could govern SushiSwap, whereas developers governed Uniswap. This distinction proved short-lived. On 16 September 2020, Uniswap's developers altered their governance plans and launched a voting rights token (UNI) [42]. Table 1 provides an overview of our selected cases.

\section{Table 1. Selected projects for a multiple-case study}

\begin{tabular}{lll}
\hline Project & Market cap* & Categorization \\
\hline Uniswap & $\$ 21,058,088,732$ & Exchange \\
Maker & $\$ 3,272,720,932$ & Lending \\
SushiSwap & $\$ 2,086,130,878$ & Exchange \\
Yearn Finance & $\$ 1,888,059,030$ & Assets \\
UMA & $\$ 1,489,888,679$ & Derivatives \\
\hline
\end{tabular}

* Data source: CoinMarketCap (14 May 2021).

Case studies are generally used to explore contemporary phenomena that are not easily distinguishable from their context [14]. For phenomena that involve a high degree of "explorability", case studies allow researchers to gather insights about motivations, to discover causal links, and to explain why singular events occurred [43]. Within the nascent and under-researched field of DeFi [1, 2, 3], we assume that the case study method is valid.

Case studies build on a combination of six evidence sources: interviews, documentation, direct observations, participant observations, archival records, and physical 
artifacts [14]. Since each DeFi case that we selected is open source, we could draw on several of these sources (Table 2). We relied on project documentation to identify differences among the cases' voting rights tokens and distribution strategies, and we used Etherscan to analyze the Ethereum blockchain and the distribution of voting rights tokens over time.

Table 2. Sources of case study data

\begin{tabular}{|c|c|}
\hline Type & Description \\
\hline (1) Documentation & $\begin{array}{l}\text { (1) } 145 \text { pages of blog entries } \\
\text { (2) } 94 \text { pages of whitepapers* }\end{array}$ \\
\hline (2) "Physical" artifact & $\begin{array}{l}\text { (1) ERC-20 tokens on the } \\
\text { Ethereum blockchain }\end{array}$ \\
\hline
\end{tabular}

* Maker and Uniswap each have a formal whitepaper. Our three other cases do not.

We adopted the two-stage process proposed by Miles et al. [44] to scrutinize each case's documentation. Firstly, we considered the documentation individually and assigned initial codes. As a team of researchers, we convened regularly to review the emerging concepts and to ensure consistency in the coding system [45]. For the second stage, we clustered codes and assigned them to higher-level themes that emerged contingently via data collection (inductive coding) or else pertained to an existing hypothesis (deductive coding). Table 3 gives a brief example of our study's deductive and inductive coding.

Table 3. Exemplary coding scheme

\begin{tabular}{|c|c|c|}
\hline Quote & $\begin{array}{l}1^{\text {st }} \text { cycle } \\
\text { coding }\end{array}$ & $\begin{array}{l}2^{\text {nd }} \text { cycle } \\
\text { coding }\end{array}$ \\
\hline $\begin{array}{l}\text { "UNI is a tradable asset } \\
\text { and functions like most } \\
\text { other standard ERC-20 } \\
\text { tokens, except it has a } \\
\text { deeper power as a } \\
\text { voting mechanism." }\end{array}$ & $\begin{array}{l}\text { UNI is an } \\
\text { ERC-20 } \\
\text { token with } \\
\text { voting } \\
\text { parameters. }\end{array}$ & $\begin{array}{l}\text { Deductive } \\
\text { Uniswap has } \\
\text { voting rights } \\
\text { tokens (UNI) }\end{array}$ \\
\hline $\begin{array}{l}\text { "Delegating UNI binds } \\
\text { the voting power of } \\
\text { your tokens to an } \\
\text { address so it may be } \\
\text { used to vote." }\end{array}$ & $\begin{array}{l}\text { Delegating } \\
\text { UNI to an } \\
\text { address } \\
\text { changes its } \\
\text { status. }\end{array}$ & $\begin{array}{l}\text { Inductive } \\
\text { Voting with } \\
\text { UNI occurs } \\
\text { on-chain }\end{array}$ \\
\hline
\end{tabular}

For the second data source, using Etherscan, we retrieved the addresses that hold any of the tokens that correspond to voting units at two separate time points: $t$ (24 May 2021) and ( $t-6)$ months. This allowed us to evaluate the case's decentrality at the present state and the change of decentrality over time. From the yielded data, we excluded automata's smart contract addresses, because automata typically do not exercise voting rights or participate in governance processes. For the cases in which voting either takes place on-chain or voting rights are staked on-chain, we excluded the addresses with balances lower than the transaction fee (gas), since they cannot afford to cast a vote. We subtracted the balance of these excluded addresses from the overall token supply. We then bundled together the balances of addresses that have the same owner (for example, exchanges like Binance, Huobi, or KuCoin) and assumed that addresses with unknown owners belong to different individuals. As a consequence of this final assumption, our analysis presents the 'best case' scenario regarding the decentrality of the voting rights tokens.

Table 4. Quantitative data collection

\begin{tabular}{lc}
\hline Case & Holders (excl. contracts and low balances) \\
\hline Uniswap & 245,162 \\
Maker & 81,254 \\
SushiSwap & 77,442 \\
Yearn Finance & 35,471 \\
UMA & 13,891 \\
\hline
\end{tabular}

We based our analysis on the number of accounts specified in Table 4 and the relevant account balances. Our conception of 'political decentralization' is based on the decentrality of voting rights tokens. To evaluate the decentrality of each case's voting rights tokens, we relied on seven metrics. For details about the seven metrics and their respective equations, please refer to Gochhayat et al. [11]. The seven metrics are:

1. Normalized Fairness (NF) [46]: A decentralized system would have higher fairness, because every participant would have equal voting power.

2. Normalized Shannon's Entropy (NSE) [47]: A centralized token distribution would have low entropy, since a few participants would heavily affect every decision.

3. Gini coefficient (G) [48]: A decentralized token distribution would have a low Gini coefficient, since most address holders would have equal voting power.

4. Cosine Similarity (CS): A decentralized token distribution would have high similarity with a system in which every address has equal voting power.

5. Jensen-Shannon Divergence (JSD) [49]: A decentralized system would have a high degree of divergence from a system in which few participants have the majority of the voting power.

6. Normalized Euclidean Distance (NED): A decentralized token distribution would have a small 
degree of distance from a system in which every address owner has equal voting power.

7. Soergel Distance (SD): The same holds as for NED.

For the sake of a casual comparison or analogy, we cited findings from Gochhayat et al. [11] about the decentrality of the "governance layer" for both Bitcoin and Ethereum. We normalized our computed metrics (or replaced the non-normalized metrics with normalized versions) to assess the values in the $[0,1]$ interval.

\section{Findings}

\subsection{Properties of voting rights tokens}

The voting rights tokens we studied are case-specific (Table 5). They have only three things in common: the tokens are all fungible and Ethereum-based (ERC-20), they grant holders the right to vote on community proposals, and they can be traded on crypto-asset exchanges. There are not many entitlements that are predetermined by developers. In a figurative sense, voting rights tokens grant holders permission to enter the Senate Floor and cast votes on the measures of the day, which are contingent and thus not pre-determined.

Table 5. Voting rights tokens' properties by case (as specified in project documentation)

\begin{tabular}{|c|c|c|c|c|c|c|}
\hline Case & 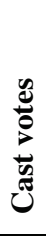 & 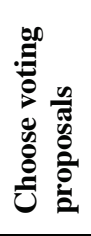 & 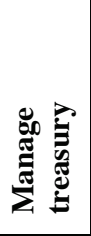 & 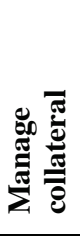 & 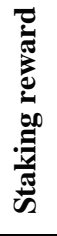 & 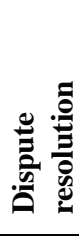 \\
\hline UNI & $X$ & $\mathrm{X}$ & $X$ & & & \\
\hline MKR & $X$ & $\mathrm{X}$ & & X & & \\
\hline SUSHI & $X$ & $X$ & & & $\mathrm{X}$ & \\
\hline YFI & $X$ & $X$ & & & & \\
\hline UMA & $X$ & & & & & $X$ \\
\hline
\end{tabular}

The projects' documentation, in total, specifies just four unique entitlements for holders of voting rights tokens: treasury management power (UNI), collateral selection power (MKR), a staking reward (xSUSHI for SUSHI holders), and dispute resolution power (UMA).

Uniswap's documentation mentions a community treasury. Uniswap's developers created the community treasury and allocated to it $43 \%$ of the total token supply (430 million UNI). The developers ceded control of the treasury to UNI token-holders on 18 October 2020 . UNI token-holders could then "vote to allocate UNI towards grants, strategic partnerships, governance initiatives, [...] and other programs" [42]. The documentation's language is purposefully unspecific, because it is not possible to predict what decisions the UNI tokenholders will make over time about the treasury funds or about particular proposals for changes to the project. Uniswap's forum participants can pose questions about the project's governance; but only UNI token-holders (and their delegates) can determine what proposals are formalized as executable code, then they can vote to implement or reject the selected proposals.

Maker's documentation specifies the ability of MKR token-holders to vote on what Ethereum-based assets are used as collateral by the lending platform. The MKR token-holders also vote on the collateral's corresponding risk parameters. (Volatile Ethereumbased assets are likely attributed strict risk parameters, whereas stable Ethereum-based assets are likely attributed lenient risk parameters.) Maker's forum participants can formulate proposals for votes, not just MKR token-holders. MKR token-holders can elect Active Proposals, then they can exercise their right to vote on the Active Proposals. The first selection process for MKR token-holders is called proposal polling; the second process is called Executive Voting.

SushiSwap's SUSHI token can be staked on the exchange. Holders then receive a tokenized derivative (xSUSHI) as a reward. The SushiSwap exchange charges participants a $0.3 \%$ trading fee. A small portion $(0.05 \%)$ of this trading fee is used to generate the xSUSHI tokens, which are then allocated to the holders of staked SUSHI tokens. At any time on the exchange, holders can convert the xSUSHI derivative to SUSHI. (The xSUSHI/SUSHI exchange rate is variable.) Only SUSHI token-holders can select what forum proposals are put up for a vote, then they can exercise their right to vote on the selected proposals.

UMA token-holders can likewise exercise their right to vote on proposals that emerge from the UMA community forum. In addition to this, UMA tokenholders have an exclusive right to cast dispute resolution votes via the UMA Voter $d A p p$. The work required to resolve disputes generates new UMA tokens (as payment for the work). The UMA token supply is thus inflationary. There are two grounds for disputes: incorrect crowd-sourced (off-chain) pricing information, and contracts liquidated for an improper reason. UMA token-holders can assess the disputes and verify the pricing data and liquidation data. UMA employs this system, named the Data Verification Mechanism, instead of an oracle's data feed. 
Yearn Finance's project documentation does not specify any unique entitlements for YFI token-holders. Forum participants can formulate proposals - any proposal whatsoever - without needing to hold YFI. YFI token-holders can then choose what proposals are put up for a vote, and they can exercise their right to vote on the selected proposals.

\subsection{Token distribution strategies}

Distribution strategies are especially important for voting rights tokens, since the token distribution determines how many individuals can exercise control over a project and how much voting power individuals possess. Our five DeFi cases all aimed for distributed governance, yet each case deployed a different token distribution strategy.

MKR tokens were pre-mined (and thus not produced by the work or stake of the lending platform's participants) and sold via an exchange. Some UNI tokens were pre-mined; others were produced by the stake of the exchange's participants. UMA's initial token supply was pre-mined, but as noted, UMA's inflationary supply is generated by dispute resolution work. Neither SUSHI nor YFI tokens were pre-mined. It follows that SushiSwap and Yearn Finance are our only two cases to deploy a fair launch strategy (which precludes pre-mined tokens).

Yearn Finance's YFI tokens must be earnt via contributions to the project (such as liquidity provision) or else purchased via crypto-asset exchanges. Since the YFI tokens were released in accordance with a strict fair launch policy, no tokens were distributed to developers. Yearn Finance's developers consequently lacked capital and could not afford to pay a third party to audit the YFI token's smart contract code. Yearn Finance's fair launch strategy thus increased the risk placed upon the community of YFI holders and offered a lack of traditional auditing guarantees. The outcome is ambiguous: the distribution of voting power incurs a distribution of risk.

SushiSwap's fair launch is also ambiguous, but its details differ notably from those of Yearn Finance's fair launch. No SUSHI tokens were pre-mined and allocated to privileged insiders like venture capitalists, but $10 \%$ of all SUSHI tokens generated by the exchange's liquidity providers automatically transferred to a treasury accessible only by developers. On 5 September 2020, a pseudonymous core developer, Chef Nomi, sold over $\$ 13$ million worth of SUSHI tokens from the SushiSwap treasury. In response to community protests and public criticism, Chef Nomi apologized on Twitter and returned the funds to the SushiSwap treasury six days later; but nothing guaranteed this outcome.

\subsection{Decentrality of the token distributions}

The decentrality of our five cases' voting rights tokens is very low. We therefore deduce that, for each of our cases, the voting power is highly concentrated.

Table 6. Measured governance decentrality of cases

\begin{tabular}{|l|l|l|l|l|l|l|l|l|}
\hline & & $\mathbf{1}-\mathbf{N F}$ & $\mathbf{1}-\mathbf{N S E}$ & $\mathbf{G}$ & $\mathbf{1}-\mathbf{C S}$ & JSD & NED & SD \\
\hline Bitcoin [11] & $\mathrm{t}=2.4 .2020$ & 0.850 & 0.315 & 0.821 & - & - & - & - \\
\hline Ethereum [11] & $\mathrm{t}=7.4 .2020$ & 0.962 & 0.438 & 0.904 & - & - & - & - \\
\hline \multirow{3}{*}{ Uniswap } & $\mathrm{t}-6$ months & 0.99974 & 0.61173 & 0.98810 & 0.98362 & 0.88687 & 0.99178 & 0.99980 \\
\cline { 2 - 9 } & $\mathrm{t}=24.5 .2021$ & 0.99980 & 0.59213 & 0.99132 & 0.98568 & 0.89963 & 0.99281 & 0.99955 \\
\hline \multirow{3}{*}{ Maker } & $\mathrm{t}-6$ months & 0.99941 & 0.57847 & 0.99491 & 0.97534 & 0.91246 & 0.98759 & 0.98757 \\
\cline { 2 - 9 } SushiSwap & $\mathrm{t}=24.5 .2021$ & 0.99938 & 0.55255 & 0.99475 & 0.97489 & 0.91257 & 0.98737 & 0.98557 \\
\hline \multirow{3}{*}{ Yearn Finance } & $\mathrm{t}-6$ months & 0.99870 & 0.46674 & 0.98724 & 0.96364 & 0.85819 & 0.98165 & 0.99999 \\
\cline { 2 - 9 } & $\mathrm{t}=24.5 .2021$ & 0.99908 & 0.46918 & 0.99098 & 0.96952 & 0.88238 & 0.98464 & 0.99999 \\
\cline { 2 - 9 } & $\mathrm{t}-6$ months & 0.99786 & 0.38747 & 0.91224 & 0.95725 & 0.64872 & 0.96148 & 0.91483 \\
\hline \multirow{3}{*}{ UMA } & $\mathrm{t}=24.5 .2021$ & 0.99892 & 0.46993 & 0.98290 & 0.96671 & 0.83568 & 0.98322 & 0.96033 \\
\cline { 2 - 8 } & $\mathrm{t}=24.5 .2021$ & 0.99921 & 0.65222 & 0.99651 & 0.97056 & 0.93797 & 0.98517 & 0.99974 \\
\hline
\end{tabular}


Table 6 presents our quantitative measurements together with the analogous measurements by Gochhayat et al. [11]. For the sake of a clearer overview, we changed the direction of the metrics NF, NSE, and CS in the table by subtracting their normalized values from 1 . As a result, high values indicate centralization.

The two cases that adopted a fair launch strategy, Yearn Finance and SushiSwap, have token distributions that are relatively less centralized than the other cases' token distributions. This aside, the distribution of YFI tokens tended towards centralization after the tokens' inception in July 2020. Likewise, the distribution of SUSHI tokens tended towards centralization after their inception in August 2020. The distribution of YFI tokens started with a greater degree of decentrality, and it remains less centralized than the distribution of SUSHI tokens. This might be caused by the $10 \%$ allocation of SUSHI tokens to the SushiSwap treasury.

UMA tokens are the most centralized. Two other cases that did not deploy a fair launch, Maker and Uniswap, are almost identical. The former's token distribution is slightly less centralized. The centralization of UNI tokens is largely attributable to the launch strategy that allocated $40 \%$ of UNI tokens to Uniswap's developers, early investors, and advisors. As of 24 May 2021, if automata's smart contract addresses are excluded, then just 23 addresses control more than $50 \%$ of the active token supply. (The active supply excludes the tokens that are held by smart contract addresses.)

\section{Discussion and outlook}

De Tocqueville's maxim about re-centralization can be rehashed for DeFi. Our multiple-case study yields a significant finding: decentralization is not actualized in the sense of evenly distributed voting power, even though decentralization is achieved in the sense of independence from centralized legal authorities and regulated companies. In political terms, hopes for evenly distributed, democratic governance are presently unfounded in our five selected cases, including the two cases that deployed a fair launch strategy. The measured distribution outcomes reveal concentrated voting power for all five cases (in comparison with a hypothetical, fully decentralized case in which each address would have equal voting rights).

In response to our findings, one cannot overlook the power of large token-holders, commonly nicknamed whales. Whales thwart the ambitions of Yearn Finance, along with other DAOs, to evenly distribute tokens and network resources [35]. Granular analysis of our data revealed that for Yearn Finance, $50 \%$ of the active token supply is controlled by just 21 accounts. These 21 accounts can potentially collude and exercise massive voting power. If they act together as a 'whale pod,' they can also manipulate token value via 'pump-and-dump' schemes [50].

Regulation limits the power of whales in traditional financial markets. Whales can swim freely by comparison in DeFi's murky waters. The unique value proposition of DeFi projects - that is, the type of decentralization that DeFi projects actually achieve - is the ability to bypass regulation and 'live' on the Ethereum network [2, 3, 36]. Ethereum's "unincorporated distributed ledger technology" has the potential to "undermine traditional forms of accountability" as well as erode the "effectiveness of traditional financial regulation and enforcement" [3]. In addition, DeFi's anonymous transactions and lack of KYC/AML checks are predictable concerns for regulators [51, 3, 52, 29, 20].

DeFi thus rehashes and intensifies the Bitcoin and Ethereum networks' history of regulatory challenges. In response, researchers and developers could turn to what Zetzsche et al. [3] describe as embedded regulation - regulators' objectives and limits hard-coded into a financial services platform. Embedded regulation could hypothetically limit the power of whales, for the sake of political decentralization. There is no guarantee, however, that embedded regulation will find success among DeFi communities and crypto-asset investors. A successful or prominent case of embedded regulation does not yet exist.

\section{Conclusion}

Non-custodial DeFi projects typically do not involve sovereign currencies, investor registration processes, or a corporate office located within a particular jurisdiction; and they are not managed or majorityowned by legal entities [3]. This motivated our exploratory, multiple-case study of five Ethereum-based DeFi projects and their tradable voting rights tokens: Uniswap, Maker, SushiSwap, Yearn Finance, and UMA. Our core finding is that each case's token distribution strategy failed to achieve measurable decentrality. In theory, voting rights tokens enable decentralized, community-based governance; but according to our measurements, the control of major DeFi projects is highly centralized.

We acknowledge the limitations of metrics used to assess decentrality as well as the fact that different metrics can yield different outcomes. We are confident, 
however, that our measured outcomes reflect reality. We employed a variety of metrics from different categories. Each metric indicated that, for all our cases, the distribution of voting rights tokens began centralized, and as time progressed, the distribution became even more centralized (Table 6). Each of the metrics we computed for our cases exhibit centralization. This means that the fair launch strategies deployed by Yearn Finance and SushiSwap achieved little success.

The nascent field of DeFi offers multiple avenues for future research. The concentrated voting power, general motives, and trading activities of DeFi whales are underresearched topics. DeFi forum posts about whales, DeFi communities' voting proposals to limit the power of whales, and DeFi commentaries by social media influencers represent valuable data sources for future social-scientific research. Legal scholars can investigate why non-custodial DeFi projects are especially challenging for regulators, and they can study developers' embedded regulation experiments.

DeFi's genuine novelty consists in the relationship between its distributed governance and regulators. What regulators face is both ironic and serious - a politically centralized yet globally diffuse form of control over multi-billion-dollar financial services. This form of control cannot be seized, limited, or broken up under current regimes.

\section{Acknowledgements}

Supported by PayPal and the Luxembourg National Research Fund FNR, Luxembourg (P17/IS/13342933/PayPal-FNR/Chair in DFS/Gilbert Fridgen).

\section{References}

[1] Amler, H., L. Eckey, S. Faust, M. Kaiser, P. Sandner, and B. Schlosser, "DeFi-ning DeFi: Challenges \& Pathway", arXiv: Cryptography \& Security, 2021.

[2] Katona, T., "Decentralized Finance: The Possibilities of a Blockchain 'Money Lego' System", Financial and Economic Review 20(1), 2021, pp. 74-102.

[3] Zetzsche, D.A., D.W. Arner, and R.P. Buckley, "Decentralized Finance", Journal of Financial Regulation 6(2), 2020, pp. 172-203.

[4] Kostakis, V., and C. Giotitsas, "The (A)Political Economy of Bitcoin”, tripleC 12(2), 2014.

[5] De Tocqueville, A., L'Ancien Régime et la Révolution, Michel-Lévy Frères, Paris, 1856.

[6] De Filippi, P., and B. Loveluck, "The invisible politics of Bitcoin: governance crisis of a decentralised infrastructure", Internet Policy Review 5(3), 2016.
[7] Hsieh, Y.-Y., J.-P. Vergne, and S. Wang, "Chapter 3: The internal and external governance of blockchain-based organizations", In M. Campbell-Verduyn, ed., Bitcoin and Beyond: Cryptocurrencies, Blockchains and Global governance. Routledge, Taylor \& Francis Group, London; New York, 2017.

[8] Reijers, W., and M. Coeckelbergh, "The Blockchain as a Narrative Technology: Investigating the Social Ontology and Normative Configurations of Cryptocurrencies", Philosophy \& Technology 31(1), 2018, pp. 103-130.

[9] Parkin, J., "The senatorial governance of Bitcoin: making (de)centralized money", Economy and Society 48(4), 2019, pp. 463-487.

[10] Schneider, N., "Decentralization: an incomplete ambition", Journal of Cultural Economy 12(4), 2019, pp. 265-285.

[11] Gochhayat, S.P., S. Shetty, R. Mukkamala, P. Foytik, G.A. Kamhoua, and L. Njilla, "Measuring Decentrality in Blockchain Based Systems", IEEE Access 8, 2020, pp. 178372-178390.

[12] Gervais, A., G.O. Karame, V. Capkun, and S. Capkun, "Is Bitcoin a Decentralized Currency?", IEEE Security \& Privacy 12(3), 2014, pp. 54-60.

[13] Oliveira, L., L. Zavolokina, I. Bauer, and G. Schwabe, "To Token or not to Token: Tools for Understanding Blockchain Tokens", International Conference of Information Systems, s.n. (2018).

[14] Yin, R.K., Case study research and applications: design and methods, SAGE, Los Angeles London New Dehli Singapore Washington DC Melbourne, 2018.

[15] Kaplan, B., and D. Duchon, "Combining Qualitative and Quantitative Methods in Information Systems Research: A Case Study", MIS Quarterly 12(4), 1988, pp. 571.

[16] Nagarajan, S., "Bitcoin hit the $\$ 1$ trillion market cap milestone twice as fast as Amazon and three times faster than Apple, data shows", Business Insider, 2021.

[17] Nakamoto, S., "Bitcoin: A Peer-to-Peer Electronic Cash System", 2008.

[18] Böhme, R., N. Christin, B. Edelman, and T. Moore, "Bitcoin: Economics, Technology, and Governance", Journal of Economic Perspectives 29(2), 2015, pp. 213238.

[19] De Filippi, P., "Bitcoin: a regulatory nightmare to a libertarian dream", Internet Policy Review 3(2), 2014, pp. 11.

[20] Foley, S., J.R. Karlsen, and T.J. Putniņš, "Sex, Drugs, and Bitcoin: How Much Illegal Activity Is Financed through Cryptocurrencies?", The Review of Financial Studies 32(5), 2019, pp. 1798-1853.

[21] Vigna, P., and M. Casey, The age of cryptocurrency: how bitcoin and the blockchain are challenging the global economic order, Picador, St. Martin's Press, New York, 2016.

[22] Pazaitis, A., P. De Filippi, and V. Kostakis, "Blockchain and value systems in the sharing economy: The illustrative case of Backfeed", Technological Forecasting and Social Change 125, 2017, pp. 105-115. 
[23] Gupta, M., and P. Gupta, "Gini Coefficient Based Wealth Distribution in the Bitcoin Network: A Case Study", In R. Sharma, A. Mantri and S. Dua, eds., Computing, Analytics and Networks. Springer Singapore, Singapore, 2018, 192-202.

[24] World Bank, "Gini Index (World Bank estimate)", The World Bank Data, 2019. https://data.worldbank.org/indicator/SI.POV.GINI

[25] Lin, Q., C. Li, X. Zhao, and X. Chen, "Measuring Decentralization in Bitcoin and Ethereum using Multiple Metrics and Granularities", 2021 IEEE 37th International Conference on Data Engineering Workshops (ICDEW), (2021), 80-87.

[26] de Vries, A., "Bitcoin's Growing Energy Problem", Joule 2(5), 2018, pp. 801-805.

[27] Drummer, D., and D. Neumann, "Is code law? Current legal and technical adoption issues and remedies for blockchain-enabled smart contracts", Journal of Information Technology 35(4), 2020, pp. 337-360.

[28] Lehmann, M., "Who Owns Bitcoin? Private Law Facing the Blockchain", European Banking Institute, 2019.

[29] Möser, M., R. Böhme, and D. Breuker, "Towards Risk Scoring of Bitcoin Transactions", In R. Böhme, M. Brenner, T. Moore and M. Smith, eds., Financial Cryptography and Data Security. Springer Berlin Heidelberg, 2014, 16-32.

[30] Buterin, V., "Ethereum: A next-generation smart contract and decentralized application platform", 2014.

[31] Buterin, V., "DAOs, DACs, DAs and More: An Incomplete Terminology Guide", Ethereum Blog, 2014.

[32] Wright, A., and P. De Filippi, "Decentralized Blockchain Technology and the Rise of Lex Cryptographia", SSRN Electronic Journal, 2015.

[33] Sunyaev, A., N. Kannengießer, R. Beck, et al., "Token Economy", Business \& Information Systems Engineering, 2021.

[34] Andersen, J.V., and C. Ingram Bogusz, "Self-Organizing in Blockchain Infrastructures: Generativity Through Shifting Objectives and Forking", Journal of the Association for Information Systems, 2019, pp. 1242 1273.

[35] DuPont, Q., "Chapter 8: Experiments in algorithmic governance - A history and ethnography of 'The DAO,' a failed decentralized autonomous organization", In Bitcoin and Beyond: Cryptocurrencies, Blockchains and Global governance. Routledge, Taylor \& Francis Group, London; New York, 2017.

[36] Chen, Y., and C. Bellavitis, "Blockchain disruption and decentralized finance: The rise of decentralized business models", Journal of Business Venturing Insights 13, 2020, pp. e00151.

[37] Young, M., "A Guide and Short History of SushiSwap", BeInCrypto, 2020.

[38] Chen, Y., "Blockchain tokens and the potential democratization of entrepreneurship and innovation", Business Horizons 61(4), 2018, pp. 567-575.
[39] Catalini, C., and J.S. Gans, "Initial Coin Offerings and the Value of Crypto Tokens", MIT Sloan Research Paper 5347(18), 2018, pp. 37.

[40] Fridgen, G., F. Regner, A. Schweizer, and N. Urbach, "Don't Slip on the Initial Coin Offering - A Taxonomy for a Blockchain enabled form of Crowdfunding", Proceedings of the 26th European Conference on Information Systems, (2018).

[41] SushiSwap, "The SushiSwap Project: An evolution of Uniswap with SUSHI tokenomics", SushiSwap Blog, 2020.

https://web.archive.org/web/20200827023308/https:/me dium.com/sushiswap/the-sushiswap-projectc4049ea9941e

[42] Uniswap, "Introducing UNI", 2020. https://uniswap.org/blog/uni/

[43] Gable, G.G., "Integrating case study and survey research methods: an example in information systems", European Journal of Information Systems 3(2), 1994, pp. 15.

[44] Miles, M.B., A.M. Huberman, and J. Saldaña, Qualitative Data Analysis: an expanded sourcebook, Sage Publications, Inc, 1994.

[45] Klein, H.K., and M.D. Myers, "A Set of Principles for Conducting and Evaluating Interpretive Field Studies in Information Systems", MIS Quarterly 23(1), 1999, pp. 28.

[46] Jain, R.K., D.-M.W. Chiu, and W.R. Hawe, "A Quantitative Measure Of Fairness And Discrimination For Resource Allocation In Shared Computer Systems", ACM Transaction on Computer Systems, 1984, pp. 39.

[47] Shannon, C.E., "A Mathematical Theory of Communication", Bell System Technical Journal 27(3), 1948, pp. 379-423.

[48] Gini, C., "On the Measure of Concentration with Special Reference to Income and Statistics", Colorade College Publication 208, 1936, pp. 73-79.

[49] Lin, J., "Divergence measures based on the Shannon entropy", IEEE Transactions on Information Theory 37(1), 1991, pp. 145-151.

[50] Kamps, J., and B. Kleinberg, "To the moon: defining and detecting cryptocurrency pump-and-dumps", Crime Science 7(1), 2018, pp. 18.

[51] Anderson, R., I. Shumailov, and M. Ahmed, "Making Bitcoin Legal”, In V. Matyáš, P. Švenda, F. Stajano, B. Christianson and J. Anderson, eds., Security Protocols XXVI. Springer International Publishing, Cham, 2018, 243-253.

[52] Renwick, R., and R. Gleasure, "Those who control the code control the rules: How different perspectives of privacy are being written into the code of blockchain systems", Journal of Information Technology, 2020, pp. 026839622094440. 